\title{
The Relevance of Cultural Sensitivity in Early Intervention
}

\author{
Emily Gardiner \\ Simon Fraser University \\ Carmel French \\ Mount Saint Vincent University
}

\begin{abstract}
This research utilized a blend of quantitative and qualitative research designs to explore the perceptions held by early interventionists regarding family-centred care, cultural diversity, and cultural sensitivity. The Executive Directors $(N=11)$ of early intervention programs in Nova Scotia completed the Cultural Diversity in Early Intervention Survey. Early intervention professionals $(N=10)$ employed in two urban programs were interviewed. Participants were asked to discuss their interpretations of family-centred care and cultural sensitivity, and to highlight any associated areas of challenge. Results demonstrated that for the most part, participants had excellent conceptual understandings of early intervention and family-centred care. Their descriptions of cultural sensitivity were less well defined. This is likely due to the fact that no participants had received training specific to cultural sensitivity and were unsupported by necessary resources, such as translators.
\end{abstract}

The nature of family-professional relationships in early intervention has changed significantly since the days when service provision was professionally-dominated in the 1950s and early 1960s. Due to the emergence of various theoretical perspectives, legislation, and supporting empirical evidence, professionals have had to reconsider their positions as the unquestioned experts and acknowledge the valuable insight families bring to intervention. Families of young children with special needs are now involved in all aspects of intervention as professionals strive to collaborate with them in family-centred ways. Employing family-centred practices with those who are culturally diverse, however, can present unique challenges for professionals.

In reviewing current early intervention literature that focuses on the core concept of family centredness (Bruder, Anderson, Schutz, \& Caldera, 1991; Trivette \& Dunst, 2005; Turnbull \& Turnbull, 1990), the importance of "cultural sensitivity" has emerged. Although current literature highlights its significance and provides general, if somewhat vague, suggestions regarding implementation, how this concept plays out in everyday practice remains unknown. As culture 
significantly influences all aspects of an individual's identity, from childrearing practices to views on disability, professionals are often unsure of how to incorporate familiar early intervention practices in the unfamiliar context of diverse values and beliefs (García Coll \& Magnuson, 2000). It has been suggested that professionals become aware of their own biases, seek culturespecific information, and attain the skills that best facilitate successful cross-cultural interactions (Chan, 1990; Lynch, 1992b). Few studies, however, have examined professionals' perceptions of such concepts. This paper explores early interventionists' perceptions of cultural sensitivity, access to services, and perceived barriers and challenges.

\section{Setting the Context}

Throughout this paper, the terms early intervention, early interventionist, cultural sensitivity, and culturally diverse families will be used frequently. It is critical that these terms are defined within the context of this research. For the purposes of this study, the definitions are as follows. With regards to early intervention, Shonkoff and Meisels' (2000) description is utilized as it captures the interdisciplinary, individualized, and family systems focus of these programs:

\footnotetext{
Multidisciplinary services provided for children from birth to 5 years of age to promote child health and well-being, enhance emerging competencies, minimize developmental delays, remediate existing or emerging disabilities, prevent functional deterioration, and promote adaptive parenting and overall family functioning. These goals are accomplished by providing individualized developmental, educational, and therapeutic services for children in conjunction with mutually planned support for their families. (pp. xvii-xviii)
}

In Nova Scotia, an early interventionist is a professional

trained to work with young children in partnership with their parents, and a variety of communitybased professionals. Under this partnership, an early interventionist prepares a developmental assessment and helps to design and implement a program to address the child's individual developmental needs. The early interventionist is knowledgeable in typical and atypical infant/early childhood development, the importance of play, family systems theory, and community based resources and programming. (Nova Scotia Department of Community Services, 2004, p. 1)

Cultural sensitivity "refer[s] to the ability of service providers to respond optimally to all children and families, understanding both the richness and limitations of the sociocultural contexts in which children and families, as well as practitioners themselves, may be operating" (Barrera \& Kramer, 1997, p. 217). Culturally diverse families refer to those whose values, practices, and beliefs differ from the mainstream culture and/or from their early interventionist. This concept extends beyond ethnic and linguistic diversity to incorporate sociocultural contexts such as "sexual orientation, economic status, work, religious beliefs, and composition” (Xu, 2007, p. 431).

\section{Review of the Literature}

\section{Evolution of the Family-Professional Partnership}

The relationships between parents of children with special needs and the professionals with whom they work have undergone significant changes over the past half-century. In the professionally-centred $1950 \mathrm{~s}$ and early $1960 \mathrm{~s}$, parental - more specifically maternal-incompetence was seen as the source of child disability, and "expert" professionals dominated interactions by determining priorities and goals without family input (Dunst, Johanson, Trivette, \& Hamby, 1991; Turnbull \& Turnbull, 1990). 
With the momentum of the 1960s civil rights reform era, legislative changes, and projects (i.e., Project Head Start) specifically aimed at helping children at risk, professionals began to utilize families in their children's intervention programs (Denholm \& Watkins, 1987). Familyallied professionals of this time taught parents how to be their child's service provider. Intervention was seen as first priority, and mothers were expected to implement programs daily (Dunst et al., 1991; Turnbull \& Turnbull, 1990).

American legislation formalizing parental involvement in the development of children's Individualized Education Plans (IEP; 1975 Education for All Handicapped Children Act) and Individualized Family Service Plans (IFSP; 1986 Education of the Handicapped Act Amendments) represented a shift in federal policy focus from the child to the family unit and corresponded with a new family-focused intervention approach (Taylor \& Baglin, 2000; United States Office of Special Education Programs, n.d.). Professionals and families now worked as teams and collaborated to identify family outcomes. Families, however, were still thought to need professional, expert advice. As programs relating to health, education, and welfare in Canada are largely controlled provincially, Canada lacks uniform national legislation regarding early intervention services for young children with, or at-risk for, developmental delays (Brynelsen \& Cummings, 1987; den Heyer \& Kienapple, 2005). As a result, "nation-wide reforms [are] much less evident in Canada [than in the United States]" (Brynelsen, Cummings, \& Gonzales, 1993, p. 165). However, it is important to note that "Canadian interest and development of services [has] paralleled the American experience" (Brynelsen et al., 1993, p. 164).

The first early intervention programs in Nova Scotia emerged in the late 1970s and were organized by parents of children with special needs and supporting community members (Nova Scotia Department of Community Services, 2004). Currently, the 21 home-based programs in the province attempt to meet the needs of children (birth to 6 years) with disabilities and their families through the use of family-centred practices. Children served by Nova Scotia's early intervention programs exhibit developmental delays of 6 months or more in two or more domains or are at risk due to the diagnoses they hold or their health histories (Nova Scotia Department of Community Services, 2008). These non-profit organizations are governed by volunteer Boards of Directors and obtain funding from the provincial Department of Community Services, Early Childhood Development Services, community partners, and fundraising efforts.

Presently, early interventionists aim to establish family-centred relationships with families (Dunst et al., 1991). In theory, professionals acknowledge the important place families hold in their children's lives and allow them to direct service delivery. A number of theoretical perspectives support the family-centred intervention philosophy including John Bowlby's (1969) attachment, Urie Bronfenbrenner's (1979) ecological, and Ann and H. Rutherford Turnbull's (1990) family systems theories. All emphasize the inextricable connection among family members and view the family unit as a dynamic system. Professionals cannot view one member (i.e., the child with disability) independently from another, as was done in the professionally-centred days of service delivery, but must take into account the needs of all members and therefore aim to benefit the entire system.

\section{Family-Centred Practices}

The main tenets of the family-centred philosophy include focusing on the entire family unit, as opposed to solely on the child; addressing families' needs, goals, and priorities; developing individualized intervention plans; and respecting families' unique strengths and capabilities. Through open and respectful communication, and by providing both formal and informal sup- 
ports in individualized and flexible ways, professionals aim to empower families, improve their overall functioning, and minimize stress (Trivette \& Dunst, 2005). In order to best address their needs, family-centred professionals attempt to acknowledge and focus their service delivery on the developmental domains that families identify as most important.

Although early interventionists strive to interact with families in empowering ways, professionals appear to struggle with implementation of recommended family-based practices (Dodd, Saggers, \& Wildy, 2009; Dunst, 2002; McWilliam, Snyder, Harbin, Porter, \& Munn, 2000; Trivette \& Dunst, 2005). Research indicates that family-professional relationships may not be collaborative, but in fact reminiscent of the past professional-as-expert approach. McBride, Brotherson, Janning, Whiddon, and Demmitt (1993) observed that in most cases, professionals alone developed children's service plans and presented them to parents for approval; families had little to no involvement in their actual development. This model neglects to acknowledge the important place of the family or to utilize their wealth of knowledge. Despite this, some families are satisfied with this role because they feel they have little to offer and much to learn (McBride et al., 1993). Others perceive this as a weakness because they would prefer to choose services that best meet their child's needs (Wehman \& Gilkerson, 1999).

Family concerns include inflexible scheduling and a focus on the child to the exclusion of other family members, both of which are in direct conflict with the central tenets of familycentred practice (Mahoney \& Bella, 1998; Wehman \& Gilkerson, 1999). Interestingly, such interventions did not result in reduced maternal stress, enhanced family functioning, or improved mother-child interactions, all of which are desirable outcomes associated with intervention (Mahoney \& Bella, 1998). These findings point to the fact that approaches that do not effectively involve the family are narrow in their perspective and have limited impact.

It is possible that this theory-practice disconnect is in part due to a lack of information about how to implement such concepts into everyday practice (Turnbull et al., 2007). For example, of the 93 articles Taylor and Baglin (2000) examined, only three addressed collaboration.

\section{Cultural Sensitivity}

Cultural sensitivity is another central component of family-centred practice that is becoming increasingly important. As the Canadian population becomes more ethnically, linguistically, and socioculturally diverse, it is inevitable that interventionists will work with families who challenge them to deliver family-centred services in new, yet culturally sensitive ways. Culture significantly impacts individuals' view and attitudes toward disability, helpseeking and childrearing behaviours, and communications styles, all of which have significant implications for family-professional partnerships (García Coll \& Magnuson, 2000; Harry, 1992; Turnbull \& Turnbull, 1990). To best meet the needs of culturally diverse families, early childhood practitioners must know how to respectfully interact with them and how to effectively incorporate their unique beliefs, practices, and values into service delivery. Research demonstrates that successful culturally sensitive programs that incorporate cultural roles and norms, native languages in sessions and handouts, and bilingual service providers, can result in improved parenting, participation, coping, and advocacy (Bruder et al., 1991; Chan, 1990).

It has been suggested that to become culturally sensitive professionals must become selfaware so they may recognize that their beliefs and behaviours are representative of only one perspective; seek culture-specific information by reading about and interacting with diverse individuals; and develop an awareness of both verbal and nonverbal cultural communication practices (Chan, 1990; Lynch, 1992a; Lynch, 1992b; Turnbull \& Turnbull, 1990). The more au- 
thentically professionals understand a family's needs, the better they will address them. The implications of culturally sensitive behaviour extend far beyond simply developing successful communications, to ultimately strengthening family competence and improving child-related outcomes. Unfortunately, researchers with the National Early Intervention Longitudinal Study (NEILS; Scarborough et al., 2004; United States Office of Special Education Programs, 2003) found that the aforementioned practices may not be employed in routine practice. Diverse families experienced less positive outcomes than those in the dominant culture, including lower satisfaction with the frequency and quality of services, and with intervention outcomes (Bailey, Scarborough, Hebbeler, Spiker, \& Mallik, 2004). This indicates that professionals are less able to meet diverse families' needs.

\section{Significance}

The aim of this research was to expand our current knowledge regarding the place of cultural sensitivity within the family-centred practice philosophy. Professionals working in early intervention programs in Nova Scotia discussed their early intervention experiences and interpretations of family-centredness, cultural diversity, and cultural sensitivity. These discussions shed light on current practices and may allow researchers and practitioners to become aware of new and positive strategies for working with culturally diverse families, as well as highlight existing areas of challenge.

\section{Method}

\section{Instrumentation}

Two measures were utilized in this study. The first, the Cultural Diversity in Early Intervention Survey, was developed by the researchers and sent to the Executive Directors of each of the early intervention programs in Nova Scotia. Questions inquired about the number of culturally diverse families served by each centre, accessible services that would support staff to serve these families, and challenges professionals had encountered in their practice.

The second measure consisted of an interview prepared by the researchers based on a review of the relevant literature and discussions with professionals currently working in the field. Open-ended, non-directive questions were utilized, allowing the researcher to discover "the interviewee's own framework of meanings" (Britten, 2006, p. 14). Interview questions encouraged participants to discuss their expectations, experiences, and perceptions surrounding early intervention and cultural sensitivity. Participants were also asked about access to necessary resources and the barriers associated with cultural diversity and early intervention.

\section{Procedure}

After obtaining the necessary university ethics approval, the Executive Directors of all early intervention programs in Nova Scotia were contacted via telephone. The research purposes, goals, and process were explained, and their participation requested. A follow-up letter outlining research aims, procedures, participant rights, and researcher responsibilities was sent to each director as well as a copy of the Cultural Diversity in Early Intervention Survey. In the second phase of the study, the Executive Directors of urban early intervention programs were asked to participate in the interview phase of the study and to distribute research packages to early interventionists employed in their programs. Each package contained (a) a letter explaining the 
research purposes and the nature of participant involvement; (b) a demographic questionnaire; (c) a consent form; and (d) a stamped and addressed return envelope. The authors' contact information was included, so those who wished to participate could either return the signed consent form or communicate with the researchers via email or telephone. Those who wished to participate contacted the first author, and a mutually agreed upon time and place was established for the interview to take place.

\section{Sample}

This study had two participant groups. The first consisted of 11 Executive Directors of early intervention programs in Nova Scotia who completed a short survey. The second group consisted of 10 early interventionists employed across two urban early intervention programs in Nova Scotia at the time of the study. It should be noted that it was also intended that culturally diverse families involved with early intervention in Nova Scotia would participate; however, as only one family made contact with their early intervention program to indicate interest in participation, this aspect of the study was dropped.

\section{Data Analysis}

The bulk of this project fits within a qualitative approach to research, in which the investigators sought to understand how and why participants had come to hold certain perspectives. The nature of the Cultural Diversity in Early Intervention Survey data, however, better leant itself to a quantitative analysis, and as such, the mean, percentages, and in some cases the range were determined. Items that demanded descriptive responses were compared qualitatively, and those cited most frequently were noted. Any findings that were unusual or went against those supported by the majority were highlighted. Such data served to supplement and contextualize interview data and are considered appropriate within the larger qualitative domain, as employing survey measures may provoke "unanticipated but valuable insights" (Grant \& Fine, 1992, p. 419). Understandings gained from interviews were deepened, and a glimpse into how the issue of cultural diversity within early intervention exists on a larger, provincial scale was provided.

Both an a priori framework and a modified grounded theory approach were utilized to analyze interview data. As questions were developed based on a review of relevant literature, they served as an initial frame for the interviews; however, other important information that arose from questions and discussions was also included. Data were analyzed using a modified grounded theory approach as the identification of themes and codes occurred inductively as they emerged from the data (Weston et al., 2001). A constant comparative approach was utilized, in which all data from emergent categories were pulled together and compared.

The interviews were transcribed verbatim and the opportunity for member checking was presented to participants, both of which serve to ensure the trustworthiness of the method (Mays \& Pope, 2006; Weston et al., 2001). The transcripts were then reviewed and initial codes were identified. Any words, phrases, or explanations that appeared to be salient were co-coded by both authors as the use of a second rater helps to eliminate bias and adds to the validity of the results. Specifically, transcripts were coded independently by each author and then brought together and compared. Differences or ambiguous statements were resolved by contextualizing within field notes. Initial codes were compared and collapsed so second level codes could be developed and data could be clustered accordingly. Third level codes and overarching themes were identified and categories were formed (Bogdan \& Biklen, 1992). This categorization process remained 
flexible to ensure that important themes could be captured and subsequently communicated. Field notes were taken during the interviews so observations of participants' body language and use of expressive gestures would be available to provide context to statements.

\section{Results}

\section{Cultural Diversity in Early Intervention Survey}

Survey results indicated that 9 of the 11 participating centres served families with culturally diverse backgrounds. Centres had a mean number of six culturally diverse families on their caseloads. The majority served between one and five, with the exception of two larger centres that served 20 and 27 diverse families. The most frequently mentioned cultures and ethnicities included First Nations/Aboriginal and African-Canadian. Other mentioned cultures included European, Asian, Arabic, Indo-Canadian, and Acadian. Seven programs indicated that they were involved with families for whom English was a second language, serving an average of four such families. Two centres indicated that translators were needed to work with their families, and three indicated that they had access to these services. It is important to note, however, that only one centre requiring translation services actually had access to them. Members of another centre stated that although they could access translators, it was done with difficulty.

Six centres indicated that having families from a diversity of cultural backgrounds presented unique challenges to early interventionists in their programs. Most frequently noted was the challenge associated with being unaware of families' differences and unique expectations. Language was the next most frequently cited challenge, with respondents indicating that it was difficult when they could not offer services in families' languages of choice. Other noted challenges included cultural perceptions of disability, finding support networks for culturally diverse and isolated families, lack of access to culturally relevant materials and resources, and a fear of inadvertently offending culturally diverse families due to their own lack of knowledge. Although four centres indicated that they were aware of supports and services for culturally diverse families of children with special needs in their area, only one indicated that they had accessed them.

Table 1

Summary of Responses to Survey Items

\begin{tabular}{|c|c|c|c|}
\hline Items & Mean & Range & Percent \\
\hline Families served by early intervention programs & 54.27 & $7-230$ & \\
\hline Centres with culturally diverse families on early intervention program caseload & & & 81.82 \\
\hline Culturally diverse families on early intervention program caseload & 5.73 & $0-27$ & \\
\hline Centres with families for whom English was a second language & & & 63.64 \\
\hline Families for whom English was a second language & 3.86 & $1-10$ & \\
\hline Centres indicating translator services are needed to work with some families & & & 18.18 \\
\hline Centres with access to translator services & & & 27.27 \\
\hline Centres indicating that culturally diverse families present unique challenges & & & 54.55 \\
\hline Centres with families who were recent immigrants to Canada & & & 36.36 \\
\hline Centres indicating awareness of supports and services for culturally diverse families & & & 36.36 \\
\hline Centres that have accessed supports and services for culturally diverse families & & & 9.10 \\
\hline Centres that do outreach for culturally diverse families & & & 9.10 \\
\hline
\end{tabular}




\section{Interview Results and Discussion}

\section{Family-Centred Practice}

Participants demonstrated a very well-developed and clear understanding of the familycentred philosophy. This was evidenced both from their explanations of what the concept of family-centred practice meant to them and how they implemented this approach into their interactions with children and families. Interviewees strongly adhered to belief systems that placed families at the centre and saw them as knowledgeable contributors who provided invaluable information about their needs and priorities. They also noted the importance of listening to families, being aware of their unique circumstances, and individualizing services accordingly. These approaches are identified in early intervention literature as being crucial to facilitating family involvement and achieving the goals of improving child and family functioning, minimizing stress, and developing confidence and competence (Beckman, 2002; Raver, 2005; Trivette \& Dunst, 2005).

Most (9 of 10) participants saw the importance of putting the family in the "driver's seat" of service delivery. This was seen as a way of acknowledging that the family knows their child best and of ensuring that their needs were accurately reflected in service plans. This philosophy is exemplified in one participant's statement: "I think it's...having a philosophy that the family are the authorities, that they know their child better than anyone else does now or ever will know their child."

Although the idea of placing the family in the expert role is widely accepted (Beckman, 2002), one professional communicated an approach that differed. She believed that it was important for service plans to reflect the goals of both families and interventionists. Despite her understanding of the fact that families are the most knowledgeable about their children, she felt it was important to "stick to some of what your beliefs are as well." This is in stark contrast to recommended practices (Trivette \& Dunst, 2005) and to what other participants communicated, as others emphasized putting aside their own priorities in favour of the family's:

[I]t's always keeping in mind what the parents' focus [is], what are their priorities, and perhaps it's not my priority...not to say that either goal is wrong...but what matters is what the family is wanting.

Research has also demonstrated, however, that many professionals struggle in conceding ultimate decision-making power to parents (McBride et al., 1993; McWilliam et al., 2000; Trivette \& Dunst, 2005). Adopting such an approach is reminiscent of the professionally-dominated days of service delivery and may point to a need for ongoing training in order to refresh interventionists about current approaches to service delivery.

When interventionists were asked how they implemented theory into practice, their responses reflected a strong understanding of the family-centred philosophy. All participants spoke about employing family-focused and responsive approaches, and about utilizing positive communication strategies. This reflects four of the five components that professionals and families have identified as important for family-centred professionals to demonstrate, including a family orientation, positivity, sensitivity, responsiveness, and friendliness (McWilliam, Tocci, \& Harbin, 1998). The final component, knowledge about child development, disabilities, and the community, was not specifically referenced in their discussions concerning family-centred practice, but was mentioned in relation to other concepts. For example, participants noted their 
understanding of child development when talking about how they got involved with early intervention, as all were educated in relevant disciplines, including Psychology and Child Studies.

This examination of participants' perceptions of family-centred practice has shown that they understand the theoretical underpinnings of the concept and believe they are incorporating them into their interactions with families. The disconnect that has been identified in previous research is also important to note, however, as an abundance of studies showcasing early interventionists' developed conceptual understanding and lacking implementation abilities exist (e.g., Mahoney \& Bella, 1998; McBride et al., 1993; McWilliam et al., 2000; Melanson, 2007; Trivette \& Dunst, 2005; Turnbull et al., 2007; Wehman \& Gilkerson, 1999). As families were not involved in this particular study, these same comparisons cannot be made.

Other researchers have suggested that this disconnect can be circumvented if interventionists clearly explain the nature of early intervention and family-centred practice to families and encourage them to take a leading role in service delivery (McBride et al., 1993; Melanson, 2007). It is important to acknowledge that when participants were asked if they thought families' early intervention expectations were met, five professionals highlighted their role in clearly explaining the above to families:

I think we need to be very clear though when we're discussing what early intervention services look like when we meet with families...what we can offer and how we can match our services to meet their family and child's need.

They felt that this not only facilitated their active involvement, but also ensured that families developed appropriate service delivery expectations, thus improving the likelihood that these would be fulfilled. Utilization of such approaches facilitates the development of an open and honest family-professional relationship, in which both can freely discuss their concerns. It is possible that this may be a distinguishing factor between early interventionists who can and cannot operate from a truly family-centred place.

The results relating to participants' perceptions of family-centred care demonstrate a number of important findings. As identified in previous research, participants in this study also appear to have a strong conceptual base of knowledge. Although one interventionist's statement led to questions regarding how well these concepts are implemented in day-to-day practice, it must be noted that the majority of participants' descriptions of their practices aligned with their conceptual understandings. The findings emphasize the necessity of including families in such studies because their valuable perspectives can provide a more developed picture of this issue and can highlight areas where change is needed.

\section{Cultural Sensitivity}

As almost all centres (9 of 11) were currently serving culturally diverse families, and all interviewed professionals had at least one family on their caseloads that they considered to be culturally diverse, interventionists' understanding of cultural sensitivity is critically important. It should be noted that professionals were the sole determinants of whether or not families were culturally diverse. It is therefore possible that families who would identify themselves as such were not recognized. In terms of how participants conceptualized diversity, one individual's description clearly portrays how the term culture extends far beyond only ethnic and linguistic difference, to include sociocultural contexts as well:

I've been working here for ten years and I haven't really worked with a whole lot of, like the first thing that comes to mind is immigrant families...I haven't worked with a lot of those families...but 
then I started thinking well but I've worked with a lot of families [who] are culturally different from me...I've worked with families where one parent was deaf... where parents maybe had intellectual impairment...with families headed by same sex couples...adoptive families, foster families, there's all kinds of different types of families that...their cultures would be different from mine...it's all kind of culture.

Chan (1990) and Lynch (1992b) discussed three critical components to becoming culturally sensitive: becoming self-aware; seeking culture-specific knowledge; and gaining the skills necessary to engage in successful interactions with diverse individuals. These were essentially reflected in participants' descriptions of the concept. The first, self-awareness, was the least represented component, as only one professional mentioned it. Although she noted the value in understanding one's own background before beginning to learn about and understand others', the ultimate purpose, which is to appreciate how one's ways of being represent only one perspective, was not mentioned (Lynch, 1992a; Turnbull \& Turnbull, 1990). Blanchett, Klingner, and Harry (2009) also suggested that professionals acknowledge that services for families of children with special needs represent "White middle-class English-speaking cultural norms and values" (p. 403). Such an understanding was not noted.

Eight participants spoke about seeking culture-specific information by asking about families' backgrounds and practices. Many thought that this served as a starting place from which relationships could be built. It also demonstrated a genuine desire to learn, an advantageous characteristic for culturally sensitive individuals to possess (Lynch, 1992b). Eight professionals also mentioned not making culture-based assumptions about what families' beliefs and practices might be:

[I]t's getting to know the family and what they believe their culture practices, because what we read in books may not be the same as they would practice...it's similar to...Canada, some things are done regional[1y]...but that's the same thing in different countries. So I find that's the best way, is get[ting] them to explain to me about their culture to make me more culture sensitive to them.

An avoidance of stereotyping based on culture-specific knowledge is another important quality of culturally sensitive professionals (Blanchett et al., 2009; Bowe, 2007).

Although early interventionists noted that they tried to be sensitive and respectful when interacting with culturally diverse families, no specific mention was made of seeking the skills that would allow them to do this. Lynch (1992b) noted that culturally sensitive individuals should observe families' unique nonverbal and verbal patterns of communication, and attempt to match their own styles of interaction to this. Practices of this sort were not mentioned.

The findings cited above demonstrate that participants' understanding of cultural sensitivity is inchoate. They professed a genuine respect for all families and an enthusiastic desire to learn. This latter aspect is of utmost importance because this will encourage a continual quest for knowledge and will further develop their understandings of the concept.

When asked how culturally sensitive practices were implemented, participants' responses focused on their overall approach. The overarching theme that emerged was openness; professionals were open to learning about different practices, to sharing personal information about their own backgrounds and beliefs, and to participating in families' traditions. Few specific culturally sensitive practices were mentioned with the exception of one interventionist who spoke about trying to integrate culturally relevant activities into her home visits and another who said she tried to learn a few words in a family's first language in order to communicate with their children. 
Chan (1990) and Bruder et al. (1991) have identified key elements that should be included in culturally sensitive programs. Interviewed professionals made no mention of having access to such services. It appears that professionals' failure to discuss specific culturally sensitive practices may be due to the fact that services and support are unavailable, as opposed to the idea that professionals are actually culturally insensitive. Their conveyed openness to and acceptance of diversity demonstrates that if they had access to such services, professionals would likely be eager to take advantage of them. The qualities of openness and acceptance should not be discredited as insufficient, as these allow families to direct service, which is a fundamental element of family-centred practice. Although professionals likely satisfy many of culturally diverse families' needs by working from such a perspective, research has demonstrated that this is insufficient as such families may experience fewer positive intervention outcomes (Bailey et al., 2004). It is therefore essential that culturally diverse families' early intervention expectations, experiences, and perceptions be obtained. This will illuminate the specific factors that these families see as missing and will better prepare professionals to satisfy an increasing demand.

An important finding is that resources to help professionals provide services in culturally sensitive ways are scarce. Across the province, only four centres were aware of supports and services for culturally diverse families of children with special needs, and only one had accessed them. This shocking statistic poignantly illustrates how great the need is for improved access to service. Access to translation services was a particularly significant issue -9 participants mentioned desiring increased access. Because interpreters are not funded, they can only be accessed through short-term, special funding, or by involving other organizations such as hospitals or schools. Interventionists instead used creative approaches-one participant mentioned using a multilingual family member. Others used translation websites, accessed fellow staff members who spoke French, and recruited other families to act as translators. One family had offered to do so; however, this service had yet to be taken advantage of. Five interventionists mentioned relying on one family member's ability to speak English:

\section{Generally if you've got a couple, parents who have little ones [who] come to be on our service, usually one of them will speak reasonably good English. Again, do we have a right to expect that? Of course not, but it is certainly very fortuitous when it happens...at least you've got one parent who can help to facilitate the service delivery.}

Problems arose, however, when this parent could not be present during visits. Forms that are filled out with parents, such as intake forms and parent surveys, are also only available in English. These represent significant barriers for both professionals and the culturally diverse families with whom they work. Providing services in families' first languages has been identified as an essential component of culturally sensitive programs (Blanchett et al., 2009; Bruder et al., 1991; Chan, 1990). At this point, early interventionists remain unable to fulfill this requirement. This may represent a serious source of risk for culturally diverse families involved in early intervention because an inability to communicate inhibits the development of an open, honest, and reciprocal family-professional partnership. It also makes it less likely that a family's needs will be met, as interventionists may not have a true understanding of what those needs are (García Coll \& Magnuson, 2000).

With regards to training, only two participants felt that they had received adequate training to prepare them to meet the needs of culturally diverse families. Interestingly, no one had received training specific to cultural diversity, awareness, or sensitivity. Although 7 participants were aware of relevant professional development sessions, only one had participated in a workshop about the immigrant experience and it was when she was employed in another profession. 
The few who felt prepared said that a combination of their personal and professional experiences had contributed to their comfort in providing culturally diverse families with a sensitive approach to service delivery. Others spoke about utilizing practices they had learned about through other training, including general sensitivity and reflectiveness training.

Although participants' application of other knowledge to the domain of cultural sensitivity demonstrates their resourcefulness, this may be an ineffective way of meeting diverse families' needs. All interview participants were currently serving at least one family whom they considered to be culturally diverse, and most felt unprepared in training and unsupported in resources to successfully do so. If early intervention programs are going to open their doors to all families, employees must be provided with the support they require. If this does not occur, service delivery for culturally diverse families is held to a lower standard. Improvements in training would improve professionals' feelings of preparedness and subsequently their abilities to serve diverse families of children with special needs.

In addition to the barriers of lack of training and supports, interview and survey participants noted communication challenges, cultural barriers, and a lack of support not only for professionals, but for families as well. For example, one participant said,

\begin{abstract}
Often these are families who are lower income...some people have left their country of origin because of living conditions and wanted a so-called better life in Canada, so sometimes, for some families that's a barrier. It's a barrier to their...day-to-day life...sometimes they're living, because of their lower income...in neighbourhoods that maybe are not as safe, may not be as nurturing, may not be as stimulating for that child...I find that these can be families that are a little bit more isolated, not only isolated by the benefit of having a child with special needs, but they're isolated because they don't know as many people, they're trying to figure out a new city potentially.
\end{abstract}

This is in accordance with Blanchett et al.'s (2009) suggestion that professionals must learn how the sociocultural contexts from which families operate can themselves act as barriers.

Participants noted how their own lack of understanding of cultural differences could be a barrier because it could lead to misunderstandings. Lynch (1992b) suggested that gaining such knowledge is crucial to becoming culturally sensitive. Participants did discuss asking families about their practices, contacting community cultural organizations, and using the Internet to further their knowledge. The latter two points demonstrate three of the desirable characteristics that culturally sensitive individuals should possess, including a sincere attempt to understand others' points of view and an openness and genuine desire to learn (Lynch, 1992b). It is important to note that professionals did not see these barriers as insurmountable and persevered to overcome them:

I try to think...I don't have to be just like you to have a beneficial relationship with you, just because I maybe look different or come from a different background, [or] use different language....I've come to see that...the situations that I've felt the most intimidated in...that I've really had to work the hardest at, have been the ones that have been the most rewarding in the end. They were the relationships that carry on after it's not a formal professional relationship anymore, so I think...there's really something to be said about just putting in the extra effort to overcome the barriers.

Participants discussed the resources they would like to see in place to assist culturally diverse families. The fact that improved access to translators was mentioned by 9 participants comes as no surprise. As language was an often-noted barrier to establishing open communication, it is understandable that professionals have a great desire for services that will amend such challenges. Of particular importance was one professional's desire to pair families with a bilin- 
gual case manager, as this is a frequently noted component of culturally sensitive programs (Blanchett et al., 2009; Bruder et al., 1991).

Other desired resources included a greater range of literature that could be used by parents and professionals, further training and professional development, and additional support services for parents, such as counselling and parenting assistance. Interestingly, three professionals wished that their staff were more culturally diverse. In reference to this, one interventionist said,

in an ideal world if we had more culturally diverse staff, it would be really neat, even the van driver, students in the playgroup, even students [on internship placements]...we're all from the same cultural background, so it would be just really neat for families to see that I think.

This component has been identified as essential to culturally sensitive programs, as professionals who share culture with families will have an implicit understanding of cultural norms and values, and therefore will be better able to incorporate them within service delivery (Blanchett et al., 2009; Bowe, 2007). Rapport development between professionals and families may be more readily established in these situations, and culturally diverse professionals may serve as more suitable role models for culturally diverse children (Bowe, 2007).

These desired supports and services demonstrate that professionals have a well-developed understanding of the kinds of supports and services that would be of great assistance to culturally diverse families of children with special needs. This serves to reemphasize the point that participating professionals aim to provide culturally sensitive services, but are unsupported by training and resources to do so. Were these supports available, professionals would likely be very adept at meeting diverse families' unique and varied needs.

\section{Limitations and Suggestions for Future Research}

The fact that interview participants involved with this study came from only two early intervention programs may present a limitation to this study. It is possible that interventionists in other centres may have worked with families who represented a more diverse range of backgrounds or who represented different cultures. As such, individuals employed in such environments may have presented different perspectives and strategies.

As all participants were from a narrow geographical area, the results obtained are unlikely to be generalizable to individuals outside of the area researched. It is important to note, however, that generalizability is not a chief concern in qualitative research and that the findings from this study still hold great value for the field of early intervention.

A final limitation concerns the fact that the findings present a uni-dimensional view as only early interventionists were interviewed. Having the perceptions of families would have facilitated a more complete understanding of the issue of cultural sensitivity and brought forth valuable suggestions for improvements to practice.

\section{Conclusion}

The purpose of this study was to ascertain early interventionists' perceptions of familycentred care and cultural sensitivity. Although this study had a relatively small participant group who came from two centres within the same municipality, it is likely that many of the identified issues are mirrored around the province. This was indicated to some extent in survey responses that were returned from early intervention programs across Nova Scotia. As such, the results 
have great value and will serve to shed light on participants' struggles in providing culturally sensitive services.

Overall, participants demonstrated strong conceptual understandings of early intervention and family-centred care that closely aligned with previous research. Although one participant made reference to a practice that conflicts with the family-centred philosophy, this finding appeared to be an anomaly because all other participants spoke about implementing practices that were family-centred in nature. The inclusion of families in similar studies is necessary if the full picture is to be garnered. Without their valuable perspectives, we cannot know if the practices and process articulated by early interventionists are actually being implemented.

When participants were asked to discuss the concept of cultural sensitivity and to describe how they implemented this into their interactions with culturally diverse families, their lack of preparation and lack of support to do so became overwhelmingly clear. Early interventionists had not received training specific to cultural sensitivity, and as a result, pulled from other knowledge to meet families' needs. Although many participants mentioned a community contact that was a great asset to them, interventionists had access to few tangible resources, most notably, translators. Eighty percent of interview participants noted that language differences often presented unique barriers when working with culturally diverse families, yet they did not have access to the services that could help to ameliorate such challenges.

The overall finding that early intervention professionals require greater training and resource support to effectively meet the needs of culturally diverse families of children with special needs is of tremendous importance. They must be supported by their profession and through government policy. Without such measures, interventionists are left to apply the "learnon-the-job" approach that emerged as common. Providing professionals with appropriate training and resources will improve their confidence and their abilities to serve diverse populations, thereby improving families' early intervention experiences and helping children reach their greatest potentials.

\section{References}

Bailey, D., Scarborough, A., Hebbeler, K., Spiker, D., \& Mallik, S. (2004). National early intervention longitudinal study: Family outcomes at the end of early intervention. Menlo Park, CA: SRI International.

Barrera, I., \& Kramer, L. (1997). From monologues to skilled dialogues: Teaching the process of crafting culturally competent early childhood environments. In P. J. Winton, J. A. McCollum, \& C. Catlett (Eds.), Reforming personnel preparation in early intervention: Issues, models and practical strategies (pp. 217-251). Baltimore, MD: Paul H. Brookes.

Beckman, P. J. (2002). Providing family-centered services. In M. L. Batshaw (Ed.), Children with disabilities (pp. 683-691). Baltimore, MD: Paul H. Brookes.

Blanchett, W. J., Klingner, J. K., \& Harry, B. (2009). The intersection of race, culture, language, and disability: Implications for urban education. Urban Education, 44, 389-409. doi:10.1177/0042085909338686

Bogdan, R. C., \& Biklen, S. K. (1992). Qualitative research for education: An introduction to theory and methods (2nd ed.). Needham Heights, MA: Allyn and Bacon.

Bowe, F. G. (2007). Early childhood special education: Birth to eight (4th ed.). Clifton Park, NY: Thomson Delmar Learning.

Bowlby, J. (1969). Attachment. In M. M. R. Khan (Ed.), Attachment and loss (Vol. 1, pp. 3-428). London, UK: The Hogarth Press.

Britten, N. (2006). Qualitative interviews. In C. Pope \& N. Mays (Eds.), Qualitative research in health care (3rd ed.; pp. 12-20). London, UK: BMJ Publishing Group.

Bronfenbrenner, U. (1979). The ecology of human development: Experiments by nature and design. Cambridge, MA: Harvard University Press. 
Bruder, M. B., Anderson, R., Schutz, G., \& Caldera, M. (1991). Niños especiales program. A culturally sensitive early intervention model. Journal of Early Intervention, 15, 268-277. doi:10.1177 $/ 105381519101500306$

Brynelsen, D., \& Cummings, H. (1987). Infant development programs: Early intervention in delayed development. In C. Denholm, R. Ferguson, \& A. Pence (Eds.), Professional child and youth care: The Canadian perspective (pp. 134-154). Vancouver, BC: University of British Columbia Press.

Brynelsen, D., Cummings, H., \& Gonzales, V. (1993). Infant development programs. In R. Ferguson, A. Pence, \& C. Denholm (Eds.), Professional child and youth care (2nd ed.; pp. 162-187). Vancouver, BC: University of British Columbia Press.

Chan, S. (1990). Early intervention with culturally diverse families of infants and toddlers with disabilities. Infants and Young Children, 3(2), 78-87. Retrieved from http://journals.lww.com/lycjournal/pages /default

den Heyer, I., \& Kienapple, K. (2005). Enhancing personnel preparation for early intervention in Nova Scotia. Task Force on Early Intervention. Halifax, NS: Mount Saint Vincent University.

Denholm, C., \& Watkins, D. (1987). Canadian school-based child care. In C. Denholm, R. Ferguson, \& A. Pence (Eds.), Professional child and youth care: The Canadian perspective (pp. 64-88). Vancouver, BC: University of British Columbia Press.

Dodd, J., Saggers, S., \& Wildy, H. (2009). Constructing the 'ideal' family for family-centred practice: Challenges for delivery. Disability \& Society, 24, 173-186. doi:10.1080/09687590802652447

Dunst, C. J. (2002). Family-centered practices: Birth through high school. Journal of Special Education, 36, 139-147. doi:10.1177/00224669020360030501

Dunst, C. J., Johanson, C., Trivette, C. M., \& Hamby, D. (1991). Family-oriented early intervention policies and practices: Family-centered or not? Exceptional Children, 58(2), 115-126. Retrieved from http:// cec.sped.org

García Coll, C., \& Magnuson, K. (2000). Cultural differences as sources of developmental vulnerabilities and resources. In J. P. Shonkoff \& S. J. Meisels (Eds.), Handbook of early childhood intervention (2nd ed.; pp. 94-114). New York, NY: Cambridge University Press.

Grant, L., \& Fine, G. A. (1992). Sociology unleashed: Creative directions in classical ethnography. In M. D. LeCompte, W. L. Millroy, \& J. Preissle (Eds.), The handbook of qualitative research in education (pp. 405-446). San Diego, CA: Academic Press.

Harry, B. (1992). Developing cultural self-awareness: The first step in values clarification for early interventionists. Topics in Early Childhood Special Education, 12, 333-350. doi:10.1177 /027112149201200306

Lynch, E. W. (1992a). From culture shock to cultural learning. In E. W. Lynch \& M. J. Hanson (Eds.), Developing cross-cultural competence: A guide for working with young children and their families (pp. 19-34). Baltimore, MD: Paul H. Brookes.

Lynch, E. W. (1992b). Developing cross-cultural competence. In E. W. Lynch \& M. J. Hanson (Eds.), Developing cross-cultural competence: A guide for working with young children and their families (pp. 35-59). Baltimore, MD: Paul H. Brookes.

Mahoney, G., \& Bella, J. M. (1998). An examination of the effects of family-centered early intervention on child and family outcomes. Topics in Early Childhood Special Education, 18, 83-94. doi:10.1177 /027112149801800204

Mays, N., \& Pope, C. (2006). Quality in qualitative health research. In C. Pope \& N. Mays (Eds.), Qualitative research in health care (3rd ed.; pp. 82-101). London, UK: BMJ Publishing Group.

McBride, S. L., Brotherson, M. J., Janning, H., Whiddon, D., \& Demmitt, A. (1993). Implementation of family-centered services: Perceptions of families and professionals. Journal of Early Intervention, 17, 414-430. doi:10.1177/105381519301700407

McWilliam, R. A., Snyder, P., Harbin, G. L., Porter, P., \& Munn, D. (2000). Professionals' and families’ perceptions of family-centered practices in infant-toddler services. Early Education \& Development, 11, 519-538. doi:10.1207/s15566935eed1104_9

McWilliam, R. A., Tocci, L., \& Harbin, G. L. (1998). Family-centered services: Service providers' discourse and behavior. Topics in Early Childhood Special Education, 18, 206-221. doi:10.1177 /027112149801800404 
Melanson, S. (2007). Family-centered practices in early intervention in Nova Scotia: Quality of life issues for families (Unpublished master's thesis). Mount Saint Vincent University, Halifax, NS.

Nova Scotia Department of Community Services. (2004). Early intervention programs in Nova Scotia: Standards and guidelines manual. Halifax, NS: Author.

Nova Scotia Department of Community Services. (2008). Early intervention program information. Retrieved from http://www.gov.ns.ca/coms/families/childcare/EarlyInterventionPrograms.html

Raver, S. A. (2005). Using family-based practices for young children with special needs in preschool programs. Childhood Education, 82(1), 9-13. Retrieved from http://www.researchconnections.org /childcare/resources/7582

Scarborough, A. A., Spiker, D., Mallik, S., Hebbeler, K. M., Bailey, D. B., Jr., \& Simeonsson, R. J. (2004). A national look at children and families entering early intervention. Council for Exceptional Children, 70(4), 469-483. Retrieved from http://www.cec.sped.org

Shonkoff, J. P., \& Meisels, S. J. (2000). Handbook of early childhood intervention (2nd ed.). New York, NY: Cambridge University Press.

Taylor, J. M., \& Baglin, C. A. (2000). Families of young children with disabilities: Perceptions in the early childhood special education literature. Infant-Toddler Intervention, 10(4), 239-257. Retrieved from http://www.worldcat.org/title/infant-toddler-intervention/oclc/22576561

Trivette, C. M., \& Dunst, C. J. (2005). DEC recommended practices: Family-based practices. In S. Sandall, M. L. Hemmeter, B. J. Smith, \& M. E. Melean (Eds.), DEC recommended practices: A comprehensive guide for practical application in early intervention/early childhood special education (pp. 107-126). Missoula, MT: DEC.

Turnbull, A. P., Summers, J. A., Turnbull, R., Brotherson, M. J., Winton, P., Roberts, R.,...Stroup-Rentier, V. (2007). Family supports and services in early intervention: A bold vision. Journal of Early Intervention, 29, 187-206. doi:10.1177/105381510702900301

Turnbull, A. P., \& Turnbull, H. R. III (1990). Families, professionals, and exceptionality: A special partnership (2nd ed.). Columbus, $\mathrm{OH}$ : Merrill Publishing Company.

United States Office of Special Education Programs. (2003). The national early intervention longitudinal study. Retrieved from http://www.sri.com/neils/

United States Office of Special Education Programs. (n.d.). History: Twenty-five years of programs in educating children with disabilities through IDEA. Retrieved from http://www.ed.gov/policy/speced/leg/ idea/history.pdf

Wehman, T., \& Gilkerson, L. (1999). Parents of young children with special needs speak out: Perceptions of early intervention services. Infant-Toddler Intervention, 9(2), 137-167. Retrieved from http://www .worldcat.org/title/infant-toddler-intervention/oclc/22576561

Weston, C., Gandell, T., Beauchamp, J., McAline, L., Wiseman, C., \& Beauchamp, C. (2001). Analyzing interview data: The development and evolution of a coding system. Qualitative Sociology, 24, 381-400. doi:10.1023/A:1010690908200

$\mathrm{Xu}, \mathrm{Y}$. (2007). Empowering culturally diverse families of young children with disabilities: The double ABCX model. Early Childhood Education Journal, 34, 431-437. doi:10.1007/s10643-006-0149-0

\section{Authors' Note}

Correspondence concerning this article should be addressed to Emily Gardiner, Department of Psychology, Simon Fraser University, RCB 5213, 8888 University Drive, Burnaby, BC, V5A 1S6. Email: emily_gardiner@sfu.ca

This research was supported by the Social Sciences and Humanities Research Council of Canada (SSHRC). The authors wish to thank the early interventionists and Executive Directors who participated in the research. 